\title{
Refocusing High-Resolution SAR Images of Complex Moving Vessels Using Co-Evolutionary Particle Swarm Optimization
}

\author{
Lei Yu ${ }^{1}{ }^{1}$, Chunsheng $\mathrm{Li}^{1}{ }^{1}$, Jie Chen ${ }^{1}$, Pengbo Wang ${ }^{1,2, *}$ and Zhirong Men ${ }^{1}$ \\ 1 School of Electronics and Information Engineering, Beihang University, Beijing 100083, China; \\ ylanlei@buaa.edu.cn (L.Y.); lics@buaa.edu.cn (C.L.); chenjie@buaa.edu.cn (J.C.); \\ menzhirong@buaa.edu.cn (Z.M.) \\ 2 Collaborative Innovation Center of Geospatial Technology, Wuhan 430079, China \\ * Correspondence: wangpb7966@buaa.edu.cn; Tel.: +86-135-2099-5334
}

Received: 30 August 2020; Accepted: 5 October 2020; Published: 11 October 2020

\begin{abstract}
To increase the global convergence and processing efficiency of particle swarm optimization (PSO) applied in the adaptive joint time-frequency, in this study an improved PSO is proposed to refocus the high-resolution SAR images of complex moving vessels in high sea states. According to the characteristics of the high-order multi-component polynomial phase signal, this algorithm provides parallel processing and co-evolution methods by setting the different permissions of the sub-population and sharing its search information. As a result, the multiple components can be extracted simultaneously. Experiments were conducted using the simulation data and Gaofen-3 (GF-3) SAR data. Results showed the processing speed increased by more than $40 \%$ and the global convergence was significantly improved. The imaging results verify the efficiency and robustness of this co-evolutionary PSO.
\end{abstract}

Keywords: synthetic aperture radar (SAR); moving vessel; multicomponent polynomial phase signal(mc-PPS); adaptive joint time-frequency (AJTF) decomposition; co-evolutionary particle swarm optimization

\section{Introduction}

Synthetic aperture radar (SAR) has the distinct ability to be able to observe vessels at all times, and is an important method in the detection and monitoring of marine moving targets [1-3]. Marine application research of SAR has been carried out around the world, and ship detection systems based on space-borne SAR have been developed and used in practical applications, e.g., the ocean monitoring workstation (OMW) system of Canada, the automated maritime surveillance tool (MaST) system of England, the Kongsberg satellite services (KSAT) system of Norway, the collect localization satellite (CLS) system of France, and the Ship Surveillance system of China. The GF-3 satellite is China's first C-band multi-polarization SAR, and has a maximum resolution of spotlight mode of $1 \mathrm{~m}$. As an ocean surveillance and monitoring satellite, it has played an increasingly important role in the field of marine theory and applications [4]. Vessels can be detected effectively using constant false alarm rate (CFAR) processing and its derivatives using high-resolution GF-3 images [5]. Wang et al. used the analytic hierarchy process by calculating the vessel's kernel density estimation, aspect ratio, and pixel number to finally obtain optimized vessel detection results [6]. Similarly, the identification of vessels can also be achieved with GF-3 SAR images using artificial intelligence techniques, such as convolutional neural networks (CNNs) and Region-CNN algorithms [7,8]. However, SAR imaging uses the relative motion of the satellite and ground targets to obtain high azimuth resolution in 
principle, assuming that the ground is static during synthetic aperture acquisition (i.e., dwell time). For a stationary vessel, the main structure of the hull surface is distinguishable in SAR images, and is easily detected and identified. On the contrary, vessels moving due to sea wind and waves appear as various distortions and are blurred (defocused) in SAR images. This can cause the shape of vessels to be distorted. As a result, the length, width, and scattering distribution cannot be accurately obtained, thus affecting the application of target detection and recognition. The motions of a marine vessel have multiple periodicities and a high degree of randomness. The different motion characteristics of each scattering point in every range cell cause different distance migration and initial phase, which result in frequency folding and wrapping in the azimuth dimension, and generate high-order non-cooperative phase errors in the SAR echo signal, ultimately reducing the precision of compensation [9]. In particular, the number of signal components in each cell increases and significant mutual interference between the components exists, which reduces the reliability of the component extraction. The SAR images of vessels are less clear, and vessels may even be unrecognizable, with worse sea conditions, longer dwell time, and higher SAR resolution. This phenomenon is frequently found in high-resolution images of space-borne SAR and airborne SAR.

Therefore, refocusing of the SAR images of vessels in complex motion has consistently been an important research subject for marine remote sensing applications. Generally, the refinement of image processing for moving SAR marine targets is divided into two main aspects: translation compensation and rotation compensation. The compensation method for translation is relatively mature, and usually use the inverse SAR (ISAR) principle and a self-focusing method, such as the phase gradient autofocus algorithm (PGA), to achieve high-quality SAR images [10]. Liu et al. [11] presented a model for numerical simulation and quantitative evaluation of the image distortions caused by each rotation of a ship. Martorella [12] applied ISAR processing to the Cosmo-SkyMed SAR system and refocused moving targets. These methods are suitable for vessels with relatively stable motion or medium-resolution SAR. However, due to the complex three-dimensional rotation in high sea states, there may be no effect of using the envelope alignment and phase correction method of ISAR processing steps. The remaining uncompensated translational and rotational terms after ISAR processing still have a large influence on the high-resolution SAR image in particular.

The time-frequency analysis method is an effective method for rotational compensation [13], and utilizes the instantaneous Doppler frequency of the target to avoid blurring. In the literature [14], the relatively stable interval of the target motion is selected for imaging using different time-frequency analysis methods. However, for high-resolution SAR, the signal of a moving vessel can be represented by a high-order multicomponent polynomial phase signal (mc-PPS), which includes complex envelope migration and Doppler wrapping. In these circumstances, the traditional time-frequency analysis methods, such as short-time Fourier transform, Wigner Ville distribution, and polynomial phase transformation [15,16], are seriously affected by cross-terms and cannot adapt to the practical applications of the mc-PPS. For an effective extraction of signal components, the adaptive joint time-frequency (AJTF) method, as an improved maximum likelihood method, is proposed to represent the mc-PPS in ISAR imaging, and offers better results without being affected by cross-terms. Li et al. [17], based on the AJFT method, estimated the phase of multiple scattering centers of ISAR data. According to the linearity relationship between the scattering point location and the Doppler change in the echo phases, refined ISAR images are achieved using the data of the best imaging period time. Searching for optimal parameter components with different extremal solutions in the solution space is a multidimensional optimization problem during AJTF processing. Therefore, optimization algorithms, such as genetic algorithms (GA) and particle swarm optimization (PSO) algorithms, are used to reduce the computing complexity of searching, and simulation results confirm the efficiency of these approaches during processing $[18,19]$. The frequency-domain extraction-based AJTF decomposition method has been proposed to deal with the high-resolution space-borne SAR. Although its feasibility and effectiveness have been verified, the imaging processing time is long and the SAR imaging results show limited improvement [1]. 
In this paper, a refocusing method is proposed to deal with the mc-PPS of the vessels' SAR data using a co-evolutionary PSO optimizing AJTF. Because the design of the method uses parallel processing, this algorithm improves the effectiveness and computing speed by extracting multiple components simultaneously. Furthermore, the convergence and global optimal ability are enhanced through the co-evolution of multiple sub-populations. The simulation high-resolution SAR data and GF-3 SAR data were processed and compared with ISAR and the classic PSO algorithm. Results show that the imaging technical indicators and image visualization were clearly improved. These results also verify the robustness and efficiency of the presented algorithm, particularly under high sea conditions.

\section{AJTF Decomposition Method}

AJTF is a global phase compensation method that has been proved to be an effective focused imaging algorithm to address the problem of non-cooperative targets [20]. This method performs parameter estimation and phase compensation, which reduces the influence of mutual interference between scattering centers and is particularly suitable for situations with different phase changes of each scattering center of the vessels in high-resolution SAR.

The echo of a moving vessel for a range cell in high-resolution SAR can be expressed in the form of the mc-PPS as follows [21]:

$$
s(t)=\sum_{m=1}^{M} A_{m} \cdot \operatorname{rect}\left[\frac{t}{T}\right] \exp \left\{j 2 \pi \sum_{n=0}^{N_{\mathrm{p}}} a_{m, n} t^{n}\right\},
$$

where $M$ is the number of components; $A_{m}$ represents the component intensity; rect $[\cdot]$ is the rectangular time window of width $T ; N_{p}$ is the polynomial order of the signal phase; $a_{m, 0}$ is a time-independent constant phase; $a_{m, 1}$ is the linear term of time $t$, which is related to the real position of the scatter point; and $a_{m, 2}$ Skimmed higher-order parameters are the phase errors generated by the target motion and need to be compensated in the SAR imaging process.

For the $m$-th component signal $s_{m}$ of the mc-PPS, the phase compensation function $s_{h}(t)$ is expressed as follows:

$$
s_{h}(t)=\operatorname{rect}\left[\frac{t}{T}\right] \exp \left\{-j 2 \pi \sum_{n=2}^{N_{\mathrm{p}}} a_{n} t^{n}\right\},
$$

By multiplying this component signal $s_{m}$ and the phase compensation function $s_{h}$, the $m$-th motion compensation is achieved. The frequency spectrum $S_{\mathrm{c}}(f)$ is obtained by applying Fourier transformation to the compensated signal, as follows:

$$
\begin{aligned}
S_{c}(f) & =F T\left\{\operatorname{rect}\left[\frac{t}{T}\right] \cdot s_{m}(t) \cdot s_{h}(t)\right\}=F T\left\{\operatorname{rect}\left[\frac{t}{T}\right] \cdot A e^{j 2 \pi a_{0}} \exp \left[j 2 \pi a_{1} t\right]\right\} \\
& =A e^{j 2 \pi a_{0}} T \sin c\left[T\left(f-a_{1}\right)\right]
\end{aligned}
$$

The objective function of the AJTF method is as follows:

$$
\left\{\begin{array}{c}
\left.\left\{\hat{a}_{h}\right\}=\underset{\mathrm{argmax}}{\operatorname{amax}}\left[\operatorname{FT}\left(s_{\mathrm{p}}(t) \cdot s_{h}(t)\right)\right]\right\} \quad, \quad n=2,3,4, \cdots, \\
\hat{a}_{1}=f_{\mathrm{p}}, \quad S_{\mathrm{c}}\left(f_{\mathrm{p}}\right)=\max \left[S_{\mathrm{c}}(f)\right]
\end{array},\right.
$$

where $\left\{\hat{a}_{n}\right\}$ refers to the estimating parameters and $f_{\mathrm{p}}$ is the peak position $S_{c \max }=S_{c}\left(f_{p}\right)$. The maximum value of the spectrum is at $f=a_{1}$. The maximum value of the spectrum is at, which is the scattering point image as the $\sin \mathrm{c}(\cdot)$ envelope function.

Through optimal estimation, the estimated signal $\widehat{s}_{m}$ of this component is obtained as:

$$
\widehat{s}_{m}(t)=\widehat{A} \cdot \exp \left\{j 2 \pi \sum_{n=1}^{N_{\mathrm{p}}} \widehat{a}_{n} t^{n}\right\},
$$


where $\widehat{A}$ is the component intensity of the maximum value of the spectrum $S_{\mathrm{c}}(f)$, and $\left\{\widehat{a}_{n}\right\}$ is the optimal estimated parameter of the signal component.

The time-domain residual signal is updated as follows:

$$
y(t)=s(t)-\widehat{s}_{m}(t)
$$

Using the same method, each signal component can be continuously estimated and extracted from the residual signal sequentially. The final estimated signal can be expressed as:

$$
\widehat{s}(t)=\sum_{m=1}^{M} \widehat{s}_{m}(t)+\mathrm{y}_{M}(t),
$$

where $M$ is the number of components, $\widehat{s}_{m}$ is the optimal estimation of the $m$-th component signal, and $y_{M}(t)$ is the residual error after extracting $M$ components.

The most critical step in AJTF decomposition processing is the search for the optimum parameters in the multidimensional solution space. Such optimal problems are generally non-convex and require substantial amounts of computation. Therefore, optimization algorithms such as PSO can be applied to AJTF decomposition, thus accelerating the speed of optimal processing and improving the global convergence ability.

\section{PSO Algorithm Applied in AJTF Decomposition}

\subsection{PSO Algorithm}

PSO is based on the movement and intelligence of swarms. This approach compares the optimization technique to bird flocking and has become an attractive alternative to other heuristic algorithms [22]. The method is initialized with a population of random solutions. Then, the bird is abstracted as a particle, which is represented by two parameters: position and velocity. The position indicates a feasible solution of the optimization and the velocity means the tendency of particles to move, which is the rate of change of the particle position. The particle tends to move to its historical optimal position and global optimal position during the iteration. The optimal state of each particle in the population during the iteration is evaluated using fitness. The method minimizes the fitness function and obtains the optima by adopting the optimum velocity of each particle toward the local and global particle. From iteration $g$ to $g+1$, the update of the velocity $\vec{V}_{i}(g+1)$ and position $\vec{P}_{i}(g+1)$ of the $i$-th particle is shown as follows:

$$
\begin{aligned}
& \vec{V}_{i}(g+1)=\omega \vec{V}_{i}(g)+r_{1} c_{1}\left(\vec{P}_{\text {Lbest }}-\vec{P}_{i}(g)\right)+r_{2} c_{2}\left(\vec{P}_{\text {Gbest }}-\vec{P}_{i}(g)\right), \quad\left(r_{1}, r_{2}\right) \in U(0,1) \\
& \vec{P}_{i}(g+1)=\vec{P}_{i}(g)+T \vec{V}_{i}(g+1)
\end{aligned}
$$

where $\omega$ is the inertial weight; $c_{1}$ and $c_{2}$ are the local and global attractive coefficients, respectively; and $\vec{P}_{\text {Lbest }}$ the $\vec{P}_{\text {Gbest }}$ represent the historical best local and global positions, respectively, in the whole swarm. $T$ is the factor of position updating. Therefore, the PSO algorithm may obtain a local optimal solution and retain multiple sub-optimal solutions. The flow chart of PSO is shown in Figure 1. 


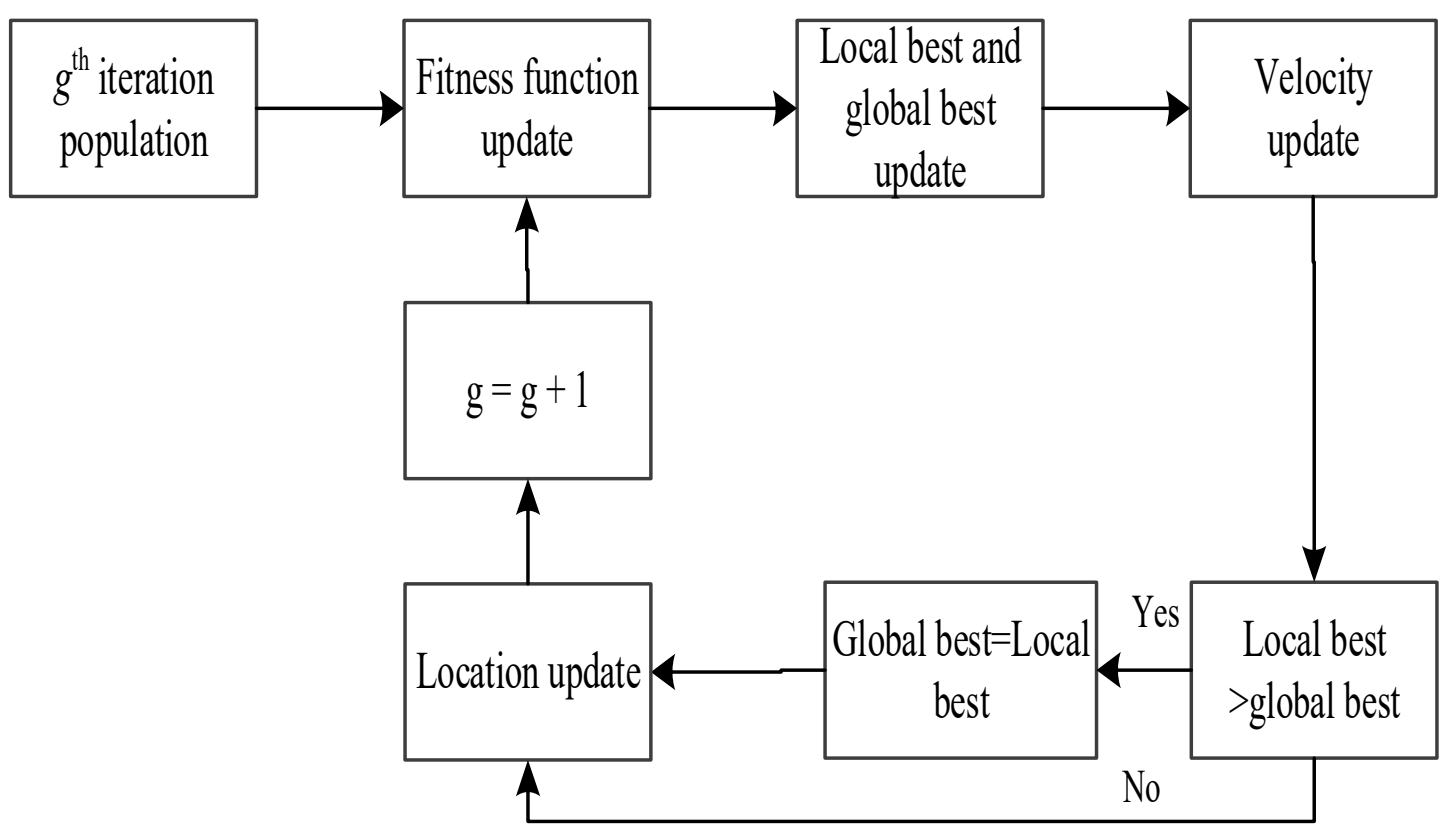

Figure 1. The flow chart of the particle swarm optimization (PSO) algorithm.

In each iteration, the position and velocity of each particle is updated, and each particle has an overall tendency to maintain its local and global best positions. The PSO algorithm retains its individual best value at the end of the algorithm. Compared with other evolutionary algorithms such as GA, PSO avoids complex genetic operations and has a population-based global search strategy. Thus, it is a more efficient search algorithm than other evolutionary algorithms [19]. Unfortunately, PSO compensation processing takes about $20 \mathrm{~min}$, which means it does not meet the requirements of the mission. However, for the mc-PPS in AJTF decomposition, PSO may obtain a locally optimal solution while retaining multiple sub-optimal solutions, which may also be true components. Therefore, PSO easily falls into the local optimal solution, which means the algorithm cannot guarantee convergence to the global optimum during the processing of the mc-PPS signal of the vessel's SAR data.

\subsection{Co-Evolutionary PSO Algorithm}

To solve the problems of the standard PSO algorithm, Shi Y. devised the co-evolutionary PSO algorithm by reducing the dimension of the fitness function. The solution is divided into multiple sub-groups, each of which is optimized by a separate algorithm, and the fitness value is evaluated and combined into a complete particle. However, the algorithm and subsequent improvements are nonetheless prone to the problem of pseudo-minimums and cannot guarantee global convergence [23]. In theory, the components of the mc-PPS are uncorrelated with each other, which indicates that the extraction of the signal components will not affect other components [18]. Therefore, the improved co-evolutionary PSO algorithm is proposed, based on the division of multiple sub-groups in the original algorithm. This algorithm sets different permissions of the sub-groups and adds a random group. It divides the entire population into several sub-groups, such as an optimal group (Opt-group), a sub-optimal group (Sub-group), and a random group (Ran-group). The term "co-evolutionary" refers to the division of groups in the solution space into several sub-groups; a sub-group represents a sub-goal to be solved, and excellent individuals found in the search migrate between different sub-groups as shared information to guide the progress of evolution. As a result, the global convergence efficiency of this algorithm is significantly improved.

The co-evolutionary PSO algorithm combines the abilities of PSO to explore the search spaces with varied priorities. The Opt-group includes the particle with the global optimal position, and the Sub-group is prohibited from iterating with the neighborhood of the Opt-group. Setting the search 
forbidden zone to the sub-optimal particle swarm strengthens the global optimal ability of the Opt-group. The Sub-group only limits the search area in the sub-optimal solutions, which means the premature stagnation state problem of the particle is solved. The Sub-group represents a sub-space in the solution space, and also represents one solution to the problem. Based on the azimuth position of the scattering center, the Opt-group and the Sub-group are distinguished and the scattering center corresponds to the maximum value of the spectrum. The random group has no best particle and is updated with the other groups, which improves the randomness of the entire population and the information exchange among the three groups. When the Opt-group falls in the local optimal solution, the Sub-group retains the ability to search for another optimal solution outside of the region. Using this approach, multiple components can be extracted simultaneously, which effectively improves the global convergence speed. The procedure flow of the co-evolutionary PSO algorithm is shown in Figure 2.

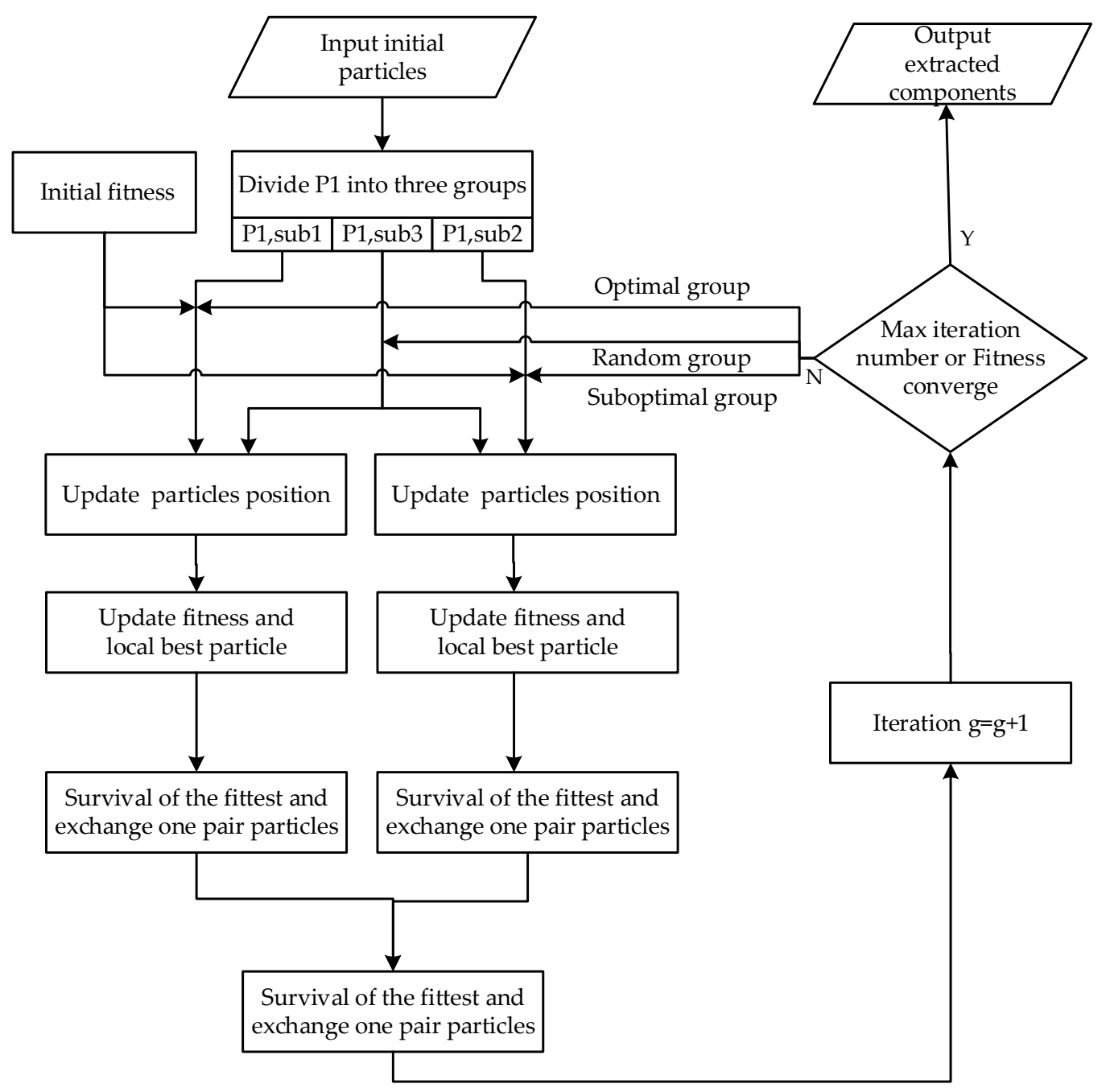

Figure 2. The procedure of the co-evolutionary PSO algorithm.

(a) The input initial particles are the residual signal of one range cell of SAR.

(b) The initial fitness. The fitness of the $m$-th particle $P_{m}$ is as follows:

$$
F_{f i t}\left(\mathrm{P}_{m}\right)=\max \left\{\left|F T\left(s_{p}(t) \cdot s_{h}\left(P_{m}, t\right)\right)\right|\right\}
$$

where $F_{f i t}\left(\mathrm{P}_{m}\right)$ is the fitness function of the $m$-th component; $F T()$ is the Fourier transform process; $s_{p}(t)$ is the signal to be processed; and $s_{h}(t)$ is the phase compensation function. The position of all 
particles refers to the individual parameters in the multidimensional solution space, including the start and end times of the signal component and the second-order and above polynomial coefficients.

$$
\vec{P}_{m}=\left\{a_{n}, \tau_{s}, \tau_{e}\right\}, m=1, \cdots, N_{p o p}, n=2,3, \cdots, N_{p},
$$

where $a_{n}$ refers to the higher-order parameters and is due to the complex movement; $\tau_{s}$ and $\tau_{\mathrm{e}}$ are the start time and end time of the time window, respectively. This indicates that the point scattering characteristics of the complex motion are variable because some signal components will appear and vanish during the dwell time. $N_{p o p}$ is the total number of particles, which mainly depends on the vessel size and SAR image resolution, and typically has a value of 50 120. $N_{p}$ is the polynomial order of the input signal phase, and has a value of $3 \sim 4$ [18].

(c) Divide $\mathrm{P} 1$ into three groups. $\mathrm{P}_{1 \text {, sub1 }}$ is the Opt-group, $\mathrm{P}_{1 \text {, sub2 }}$ is the Sub-group, and $\mathrm{P}_{1 \text {, sub3 }}$ is the Ran-group. Iteration is set to $g=1$.

(d) Update particle position. By random division, the Ran-group is divided into two sub-groups and combined with the Opt-group and Sub-group in two new mixed groups.

(e) Update fitness and local best particles. According to the fitness function, the global optimal and sub-optimal particle fitness are searched for in the respective ranges, and the optimal particle parameters and scattering center positions are updated. Observing the near zone of the Opt-group scattering center is prohibited to the Sub-group, which has a value of 20 30.

In the $g$-th iteration, the local and global optimal fitness values of the $m$-th particle are as follows:

$$
\left\{\begin{array}{l}
F_{\mathrm{L}, g, m}=\max \left\{F_{\mathrm{fit}}\left(\left\{\vec{P}_{m}\left(g^{\prime}\right)\right\}\right)\right\}, g^{\prime}=1,2, \cdots, g, \\
F_{\mathrm{G}, g}=\max \left\{F_{\mathrm{L}, g^{\prime}, m}\right\}
\end{array}\right.
$$

where $\vec{P}_{m}\left(g^{\prime}\right)$ represents all position records from the 1st iteration to the $g$-th iteration of the $m$-th particle. Correspondingly, the local and global optimal positions of the $m$-th particle are as follows:

$$
\left\{\begin{array}{c}
\vec{P}_{\text {Lbest }}(g)=\operatorname{argmax}\left\{F_{\mathrm{L}, g, m}\right\} \\
\vec{P}_{\text {Gbest }}(g)=\operatorname{argmax}\left\{F_{\mathrm{G}, g}\right\}
\end{array}, m=1,2, \cdots, N_{\text {pop }},\right.
$$

(f) According to local fitness results in the new mixed group of the Opt-group, the survival of the fittest is made by exchanging the two particles inside if the maximum fitness value of the random Sub-group is more than the minimum value of the Opt-group. The same processing is applied to the other mixed group of the Sub-group.

(g) Similarly, according to global fitness results, the survival of the fittest is made by exchanging the roles of the two groups if the maximum fitness value of the Sub-group is more than that of the Opt-group.

(h) The iteration $g=g+1$. Based on the previous two steps, the new groups become the $(g+1)$-th population. The local best position is updated as follows. Furthermore, the global best position can also be obtained, according to Formula (12). When the fitness is stable, the update velocities of the optimal particle become zero, and the position, which is equivalent to the global optimal position, no longer changes.

$$
\vec{P}_{\text {Lbest }}(g+1)=\left\{\begin{array}{l}
\vec{P}_{m}(g+1), \quad F_{\text {fit }}\left(\vec{P}_{m}(g+1)\right) \geq F_{\text {fit }}\left(\vec{P}_{\text {Lbest }}(g)\right) \\
\vec{P}_{\text {Lbest }}(g) \text { otherwise }
\end{array}\right.
$$

(i) Whether the stop conditions are met is determined, including the maximum iteration number and the convergence of the global fitness results. The maximum iteration number is generally 300 , and the convergence is expressed by the fitness ratio of the residual signal of the sub-optimal solution 
before and after the extraction of the global optical component, which is usually set to 0.99 . If the conditions are met, step (j) is performed, and if not, then step (e) is performed.

(j) Finally, the global best parameters of Opt-group and Sub-groups are outputted, and the algorithm of one range cell is completed and it continues to process the next range cell. When the traversing of all range cells is completed, the two-dimensional imaging result is obtained with the AJTF decomposition method [1].

\section{Experiment Results and Analysis}

\subsection{Simulation Test}

The modeling and simulation system of space-borne SAR was established. The vessel scattering model consists of nine points on the edge of the hull. The sea condition of the complex motion was Level 5 [24]. The main simulation parameters of the moving vessel and imaging condition of the GF-3 satellite are shown in Table 1.

Table 1. Main simulation parameters.

\begin{tabular}{cc}
\hline Item & Value \\
\hline Scattering point model of the vessel with 9 points & {$[00,1000,-1000$,} \\
{$[X, Y]$} & $5050,500,50-50$, \\
Vessel rotation amplitude & $50,-500,-50-50][\mathrm{m}]$ \\
Vessel rotation period & {$[38.4,3.4,3.8][\mathrm{deg}]$} \\
[Roll, Pitch, Yaw] & {$[12.2,6.7,14.2][\mathrm{s}]$} \\
Looking Angle & $31.2[\mathrm{deg}]$ \\
Imaging Mode & Spotlight \\
Band Width & $240[\mathrm{MHz}]$ \\
PRF & $3725.6[\mathrm{~Hz}]$ \\
Sample Rate & $266.667[\mathrm{MHz}]$ \\
Aperture Time & $4.0[\mathrm{~s}]$ \\
Shortest Slant Range & $898.388[\mathrm{Km}]$ \\
\hline
\end{tabular}

To evaluate the performance, the classical PSO and the proposed algorithm applied to AJTF were compared in the simulation experiment. The imaging results of the scattering point model simulation are shown in Figure 3. Figure $3 \mathrm{a}, \mathrm{b}$ shows the results of the stationary vessel and the moving vessel, respectively, which were obtained using the Chirp Scaling (CS) algorithm of the conventional SAR ground processing system. Significant differences exist between the results of the model with nine scattering points. Under the stationary condition, all of the points are similar; moreover, the intensity value of the points is also relatively larger, indicating that the focusing effect was better. On the contrary, the imaging result of the vessel in complex motion is defocused and fuzzy, and it is not possible to effectively identify the points of the simulation model. The exception is the central point, which remains focused in the image because it is the center of rotation and is thus not influenced by the rotational motion. The eight surrounding points are distorted and differ from each other. These differences are due to the phase error caused by the rotation movement and the different positions. Figure $3 c$,d shows the results of the moving vessel using the classical PSO and co-evolutionary PSO, respectively. During the PSO processing, the number of iterations is 300 , and the number of particles is 100. The scattering points are focused better than those using the CS algorithm. The nine scattering points of the modeled vessel are obvious and identifiable. The focusing effect in the azimuth (Figure $3 \mathrm{~d}$ ) is more obvious in the detail of its points, which indicates that complex moving compensation is more accurate compared with that using classical PSO. 


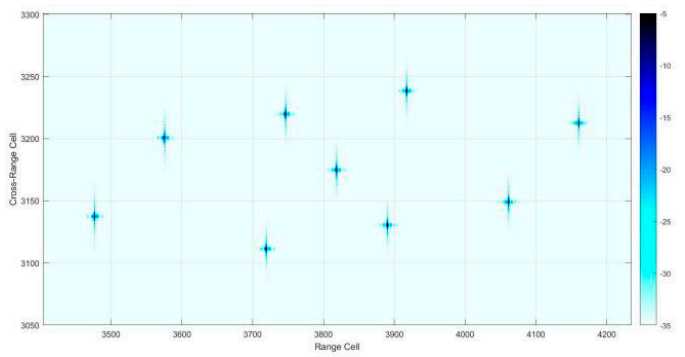

(a)

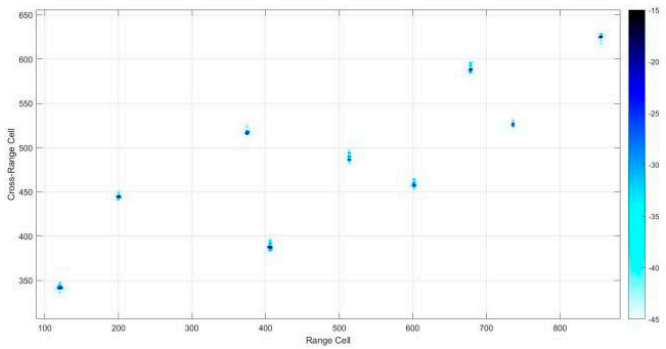

(c)

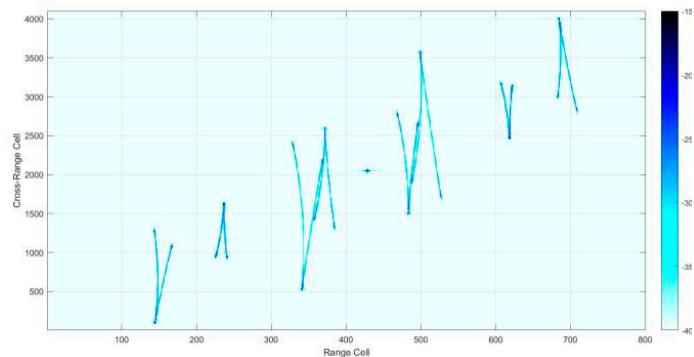

(b)

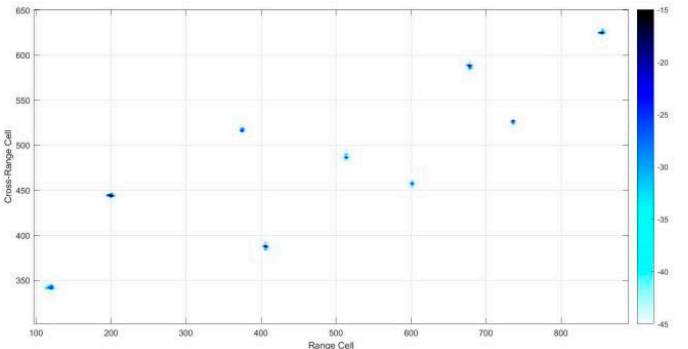

(d)

Figure 3. Imaging results of the vessel scattering point model: (a) imaging result of a simulated stationary vessel; (b) imaging result of a simulated moving vessel; (c) imaging result of classical PSO; (d) imaging result of co-evolutionary PSO.

To evaluate the effects of point refocus, the peak sidelobe ratio (PSLR) and the integral sidelobe ratio (ISLR) in the azimuth were calculated from the eight surrounding scattering points, and are shown in Table 2. Compared to the imaging results, the PSLR and ISLR of co-evolutionary PSO were superior. The improvement of the average azimuth PSLR was greater than $1.45 \mathrm{~dB}$, and the average azimuth ISLR improvement was greater than $2.1 \mathrm{~dB}$, compared to classical PSO in this simulation test. This indicates the compensation accuracy of the high-order phase error is higher and the algorithm is more robust. Finally, the processing speed of co-evolutionary PSO is about $48.97 \%$ faster than that of classical PSO, indicating a significant improvement in the global convergence. Computational complexity relates mainly to iterative processing, including the fitness update and individual parameter update. The fitness update requires the Fourier transform, and the number of calculations in the extracting components is related to the number of population individuals and the total number of iterations. Unfortunately, the PSLR and ISLR in the subsequent experiment were difficult to measure due to the clustered scattering points of the vessel in the real SAR data.

Table 2. Comparison of the algorithms.

\begin{tabular}{cccc}
\hline & CS & Classical PSO & Co-Evolutionary PSO \\
\hline PSLR (dB) & -5.02 & -10.12 & -11.57 \\
ISLR (dB) & -5.41 & -13.55 & -15.65 \\
Ave computation time $(\mathrm{s})$ & 65 & 1421 & 725 \\
\hline
\end{tabular}

\subsection{Experimental Test}

To evaluate the imaging effect and usability of this algorithm, actual GF-3 SAR stationary and moving vessel data were used to verify the experiment, as shown in Figure 4. According to the imaging time and location of the moving vessel in a high sea state taken from the GF-3 satellite, we determined that the wave height was $2.8 \mathrm{~m}$ and the ocean velocity was $1.42 \mathrm{~m} / \mathrm{s}$ at the time the image was captured [25]. In addition, the stationary vessel was selected at other times near the area in a calm sea state to compare the effects of complex motion on SAR imaging. Figure $4 a, b$ show the results of the stationary and moving vessels using the CS algorithm. Figure 4c shows the refocusing result of 
the moving vessel using ISAR processing algorithms [26]. Figure 4d,e show the imaging results of the moving vessel using the classical PSO and co-evolutionary PSO algorithms, respectively.

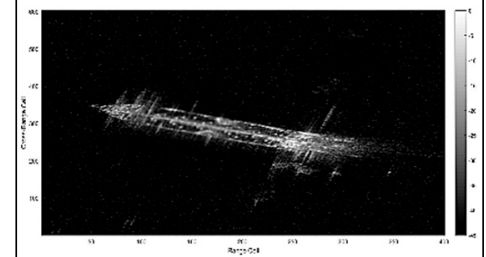

(a)

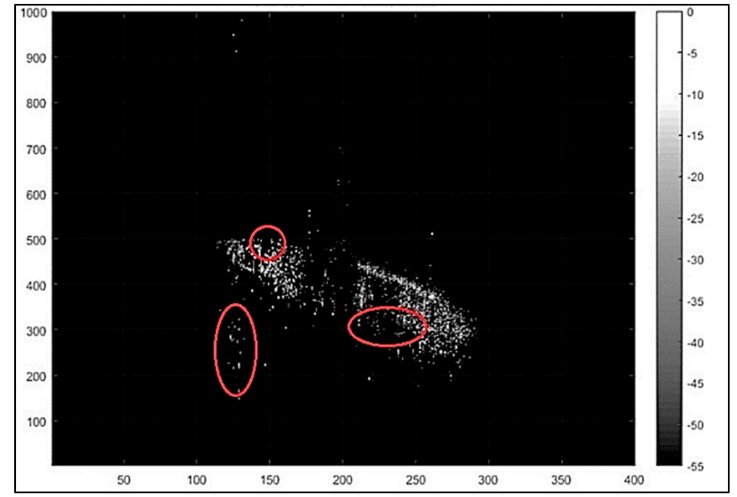

(d)

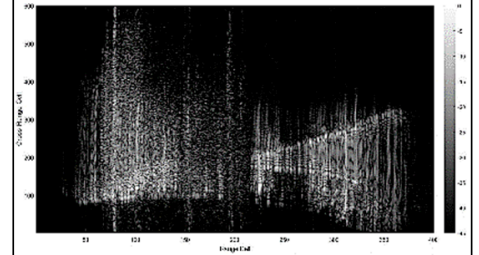

(b)

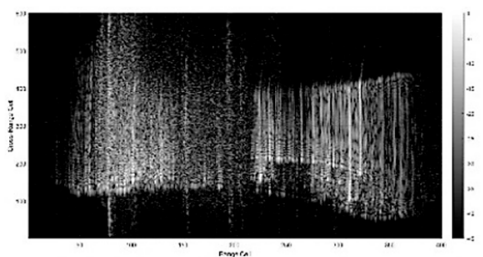

(c)

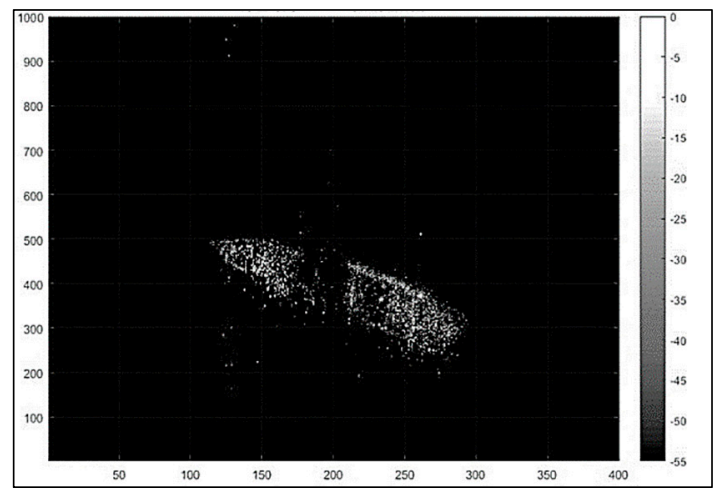

(e)

Figure 4. Imaging results of the GF-3 data: (a) imaging result of stationary vessel; (b) imaging result of moving vessel; (c) imaging result of the inverse synthetic aperture radar (ISAR) process; (d) imaging result of classical PSO; (e) imaging result of co-evolutionary PSO.

In Figure $4 \mathrm{a}$, the edge of the stationary vessel is clear and the inside structure is easy to identify on the calm sea, similar to the case of the SAR imaging result of a stationary ground target. However, as shown in Figure 4b, the vessel image in a high sea state is highly unfocused, and it is impossible to measure the parameters of the vessel and recognize the vessel type. The ISAR principle and the phase gradient autofocus method were used to accomplish the phase compensation of the moving vessel, shown in Figure 4c. Compared with Figure $4 \mathrm{~b}$, the focus in the azimuth is improved, indicating that there is a translation component of the vessel. However, previous research has shown that the ISAR method is only applicable to vessels with stable motion. Due to the different movements of the various parts of the vessel, this method is not able to provide complete compensation. Residual uncompensated translation and rotation errors after ISAR processing still have a significant influence on the SAR image. Figure $4 \mathrm{~d}$,e shows that the information of the scattering centers of each range unit is effectively extracted. The basic outline of the vessel is maintained, and the length and width of the vessel can be easily measured. In addition, the resolution of each scattering center is improved and the imaging blurring problem caused by phase wrapping is resolved. The edge shape of the vessel in Figure 4e shows more image detail than that of $4 \mathrm{~d}$; however, many noise spots are present in the red circles of the classical PSO result image, indicating that it may have fallen into a local optimal solution, and the stability and reliability of the classical PSO algorithm are comparatively worse. In addition, the processing time of co-evolutionary PSO was about $10 \mathrm{~min}$, representing a reduction of about $42.2 \%$ compared to classical PSO, which is consistent with the simulation results. Hence, the results proved the efficiency and robustness of this co-evolutionary PSO algorithm by simulation and experimental testing.

\section{Conclusions}

According to the relevant statistics, the number of defocused vessel images affected by complex motion accounts for $15-20 \%$ of GF-3 high-resolution ocean data. The SAR echo of vessels subject to 
complex motion is a multicomponent polynomial phase signal, which leads to the inability of classical time-frequency analysis methods to process data efficiently. Therefore, a novel co-evolutionary PSO applied to AJTF is proposed that can extract several components in the solution space simultaneously and avoid falling into a local optimal solution. Compared with other algorithms, this method has obvious advantages in image focusing performance, robustness, and efficiency. The results of simulation data and GF-3 satellite SAR data show that the image of a moving vessel in a high sea state was improved and, simultaneously, the processing speed of the algorithm was increased by over $40 \%$. According to our preliminary experiments, this algorithm is also suitable for sub-meter space-borne SAR and airborne SAR data processing, with a processing time of several hours. The existing method performs compensations after traditional SAR imaging and involves redundant calculations. Therefore, we will continue to study methods for simultaneous image processing and phase estimation compensation to improve computing efficiency for processing sub-meter resolution SAR data. To summarize, the use of the proposed and ISAR methods [26] in existing GF-3 SAR ground processing systems could have significant benefits for marine users who need high-precision images of moving marine vessels in applications such as vessel identification and intelligent feature extraction.

Author Contributions: Conceptualization C.L. and J.C.; methodology: L.Y. and P.W.; writing-original draft preparation, L.Y.; writing-review and editing, P.W. and Z.M.; funding acquisition, C.L. All authors have read and agreed to the published version of the manuscript.

Funding: This research received no external funding.

Conflicts of Interest: The authors declare no conflict of interest.

\section{References}

1. Lao, G.; Yin, C.; Ye, W.; Sun, Y.; Li, G.; Han, L. An SAR-ISAR Hybrid Imaging Method for Ship Targets Based on FDE-AJTF Decomposition. Electronics 2018, 7, 46. [CrossRef]

2. Crisp, D.J. The State-of-the-Art in Ship Detection in Synthetic Aperture Radar Imagery; Defence Science and Technology Organisation: Edinburgh, Australia, 2004.

3. Gao, G.; Liu, L.; Zhao, L.; Shi, G.; Kuang, G. An adaptive and fast CFAR algorithm based on automatic censoring for target detection in high-resolution SAR images. IEEE Trans. Geosci. Remote Sens. 2008, 47, 1685-1697. [CrossRef]

4. Lin, M.; He, X.; Jia, Y.; Bai, Y.; Ye, X.; Gong, F. Advances in marine satellite remote sensing technology in China. Haiyang Xuebao 2019, 41, 99-112.

5. Hwang, S.I.; Ouchi, K. On a novel approach using MLCC and CFAR for the improvement of ship detection by synthetic aperture radar. IEEE Geosci. Remote Sens. Lett. 2010, 7, 391-395. [CrossRef]

6. Wang, C.; Zhang, H.; Wu, F.; Jiang, S.; Zhang, B.; Tang, Y. A Novel Hierarchical Ship Classifier for COSMO-SkyMed SAR Data. IEEE Geosci. Remote Sens. Lett. 2014, 11, 484-488. [CrossRef]

7. An, Q.; Pan, Z.; You, H. Ship detection in Gaofen-3 SAR images based on sea clutter distribution analysis and deep convolutional neural network. Sensors 2018, 18, 334. [CrossRef]

8. Kang, M.; Leng, X.; Lin, Z.; Ji, K. A modified faster R-CNN based on CFAR algorithm for SAR ship detection. In Proceedings of the 2017 International Workshop on Remote Sensing with Intelligent Processing (RSIP), Shanghai, China, 18-21 May 2017; pp. 1-4.

9. Yu, L.; Li, C.; Wang, P.; Fang, Y. Imaging of maritime rotation targets in spaceborne SAR image. In Proceedings of the 2017 Progress in Electromagnetics Research Symposium-Fall (PIERS-FALL), Singapore, 19-22 November 2017; pp. 503-507.

10. Noviello, C.; Fornaro, G.; Martorella, M.; Reale, D. ISAR add-on for focusing moving targets in very high resolution spaceborne SAR data. In Proceedings of the 2014 IEEE Geoscience and Remote Sensing Symposium, Quebec City, QC, Canada, 13-18 July 2014; pp. 926-929.

11. Liu, P.; Jin, Y.-Q. A study of ship rotation effects on SAR image. IEEE Trans. Geosci. Remote Sens. 2017, 55, 3132-3144. [CrossRef]

12. Martorella, M.; Pastina, D.; Berizzi, F.; Lombardo, P. Spaceborne radar imaging of maritime moving targets with the Cosmo-SkyMed SAR system. IEEE J. Sel. Top. Appl. Earth Obs. Remote Sens. 2014, 7, 2797-2810. [CrossRef] 
13. Lu, G.; Bao, Z. Range instantaneous Doppler algorithm in ISAR based on instant frequency estimation. J. Xidian Univ. 1998, 3545, 198-201.

14. Hu, C.; Ferro-Famil, L.; Kuang, G. Ship discrimination using polarimetric SAR data and coherent time-frequency analysis. Remote Sens. 2013, 5, 6899-6920. [CrossRef]

15. Djurović, I.; Simeunović, M. Review of the quasi-maximum likelihood estimator for polynomial phase signals. Digit. Signal Process. 2018, 72, 59-74.

16. Djurović, I.; Simeunović, M.; Wang, P. Cubic phase function: A simple solution to polynomial phase signal analysis. Signal Process. 2017, 135, 48-66. [CrossRef]

17. Li, J.; Hao, L.; Chen, V. An Algorithm to Detect the Presence of 3D Target Motion from ISAR Data. Multidimens. Syst. Signal Process. 2003, 14, 223-240. [CrossRef]

18. Yu, L.; Lao, G.; Li, C.; Sun, Y.; Li, Y. A Novel Multicomponent PSO Algorithm Applied in FDE-AJTF Decomposition. Electronics 2019, 8, 51. [CrossRef]

19. Brinkman, W.; Thayaparan, T. Focusing ISAR images using the AJTF optimized with the GA and the PSO algorithm-comparison and results. In Proceedings of the 2006 IEEE Conference on Radar, Verona, NY, USA, 24-27 April 2006; p. 8.

20. Li, Y.; Fu, Y.; Li, X.; Le-Wei, L. ISAR imaging of multiple targets using particle swarm optimization-adaptive joint time-frequency approach. IET Signal Process. 2010, 4, 343-351. [CrossRef]

21. Liu, Q.Y.; Li, Z.S.; Li, H.Y.; Liang, H. Parameter estimation of multicomponent Polynomial Phase Signal. Acta Electronic. Sinica. 2004, 12, 2031-2034.

22. Shi, Y.; Eberhart, R. A Modified Particle Swarm Optimizer. In Proceedings of the 1998 IEEE International Conference on Evolutionary Computation Proceedings. IEEE World Congress on Computational Intelligence (Cat. No.98TH8360), Anchorage, AK, USA, 4-9 May 1998.

23. Shi, Y.; Krohling, R.A. Co-evolutionary particle swarm optimization to solve min-max problems. In Proceedings of the 2002 Congress on Evolutionary Computation CEC'02 (Cat. No.02TH8600), Honolulu, HI, USA, 12-17 May 2002.

24. Wehner, D.R. High Resolution Radar; Artech House, Inc.: Norwood, MA, USA, 1987; p. 484.

25. Global Weather Visual Simulation Network. Available online: https://earth.nullschool.net (accessed on 10 September 2019).

26. Yu, L.; Li, C.; Wang, P.; Fang, Y.; Feng, X. Research into SAR imaging of moving ship target based on ISAR technology. In Proceedings of the International Geoscience and Remote Sensing Symposium (IGARSS 2018), Valencia, Spain, 22-27 July 2018; pp. 2290-2293.

(C) 2020 by the authors. Licensee MDPI, Basel, Switzerland. This article is an open access article distributed under the terms and conditions of the Creative Commons Attribution (CC BY) license (http://creativecommons.org/licenses/by/4.0/). 